\title{
Problemas de liderazgo en la alta dirección de instituciones de salud
}

\author{
Leadership problems in the management of health institutions
}

\author{
Nilson A. Contreras-Carreto ${ }^{*}$ y Martha L. Ramírez-Montiel \\ ${ }^{1}$ Dirección General de Calidad y Educación en Salud, Secretaría de Salud, Ciudad de México; ${ }^{2}$ Clínica de la Mujer, Hospital Regional de Alta
} Especialidad Ixtapaluca, Secretaría de Salud, Ixtapaluca, Estado de México. México

\section{Resumen}

En las instituciones de salud es frecuente que los médicos u otros profesionales de la salud ocupen altos cargos directivos, habitualmente sin ninguna experiencia administrativa, de tal modo que un excelente clínico se puede convertir en un pésimo líder y administrador. En este artículo se abordan las consecuencias administrativas y organizativas de un mal ejercicio del liderazgo, interpretando el fenómeno desde las teorías clásicas y contemporáneas del liderazgo y exponiendo los problemas que la alta dirección en salud enfrenta frecuentemente en los hospitales. El liderazgo no es innato, es una habilidad que puede ser desarrollada. Liderar y administrar instituciones de salud es una ciencia y un arte que bien debiese aprenderse desde el pregrado y perfeccionarse en el posgrado, puesto que, a menos que el profesional de la salud posea una sólida carrera administrativa y política, el ocupar altos cargos de responsabilidad es un asunto fortuito.

Palabras clave: Administración. Dirección hospitalaria. Liderazgo.

\begin{abstract}
In health institutions, physicians or other health professionals frequently occupy senior management positions, usually without any administrative expertise. In such a way that an excellent clinician can become a lousy leader and administrator. In this article, the administrative and organizational consequences of a bad exercise of leadership are displayed, interpreting the phenomenon from the classical and contemporary theories of leadership and exposing the problems that top management in health frequently faces in hospitals. Leadership is not innate; it is a skill that can be developed. Leading and managing health institutions is a science and an art that should be learned from undergraduate and perfected in the postgraduate course. Unless the health professional has a solid administrative and political career, holding senior positions in the hospital is a fortuitous matter.
\end{abstract}

Key words: Administration. Hospital management. Leadership.

\section{Correspondencia:}

*Nilson A. Contreras-Carreto

Avda. Marina Nacional, 60

Col. Tacuba, Alcaldía Miguel Hidalgo

C.P. 11410, Ciudad de México, México

Fecha de recepción: 04-07-2019

Cir Cir. 2020;88(4):526-532

E-mail: itzmard@yahoo.com.mx

Fecha de aceptación: 17-10-2019

DOI: $10.24875 / C I R U .19001413$

Contents available at PubMed

www.cirugiaycirujanos.com

0009-7411/@ 2019 Academia Mexicana de Cirugía. Publicado por Permanyer. Este es un artículo open access bajo la licencia CC BY-NC-ND (http://creativecommons.org/licenses/by-nc-nd/4.0/). 


\section{Introducción}

Durante la vida laboral del médico (o de cualquier otro profesional de la salud) puede presentarse, incluso a veces sin planearse, la oportunidad de ocupar altos puestos directivos en alguna institución de asistencia en salud (centros de salud, clínicas, hospitales, jurisdicciones sanitarias, etc.), de tal manera que el profesional clínico, capacitado integralmente para la prevención, el diagnóstico, el tratamiento y la rehabilitación del paciente, se enfrenta al desafío de liderar instituciones de salud sin las habilidades necesarias para el ejercicio de sus funciones administrativas. De este modo, un excelente clínico puede convertirse en un pésimo administrador.

Si se considera la premisa de que el liderazgo es uno de los principales factores de éxito en una organización ${ }^{1}$, la crisis actual en las instituciones de salud podría entenderse como un problema secundario al mal ejercicio del liderazgo o incluso a la ausencia de este. Aún en la actualidad existen pocas investigaciones centradas en estudiar el liderazgo aplicado a instituciones de salud. En su gran mayoría, las publicaciones presentan exclusivamente casos de éxito, sin profundizar en la posible mala praxis de la alta dirección de instituciones de salud (ADIS). Por tal motivo, es necesario realizar un análisis de las diversas instituciones de salud como sujeto de estudio y analizar las características de la ADIS como objeto de estudio, así como los problemas de liderazgo que enfrenta, mediante el análisis del fenómeno desde las teorías clásicas y contemporáneas del liderazgo.

\section{Las teorías clásicas y contemporáneas del liderazgo}

Desde que el hombre vive en sociedad se ha servido del liderazgo para el cumplimiento de metas comunes. Su estudio ha interesado a diversas disciplinas, como la administración, la psicología, la sociología y la antropología, entre otras. Por tal motivo, las teorías propuestas son diversas y con diferentes enfoques, pero más que como divergentes deben entenderse como complementarias entre sí. Algunas describen cuáles son las características que hacen de un directivo un buen líder; otras, los tipos de conducta necesaria para lograr un liderazgo efectivo².

Una de las principales teorías clásicas es la teoría de los rasgos, la cual afirma que los líderes poseen rasgos innatos específicos y universales que los hacen efectivos. Estos rasgos pueden ser físicos, de personalidad, inteligencia, capacidad para la habilidad social, o relacionados con la tarea y las capacidades administrativas. La teoría también hace mención al carisma, la seguridad en sí mismo, la asertividad, la habilidad comunicativa, la creatividad y la innovación. Sin embargo, no existe suficiente evidencia para asegurar que poseer estos rasgos asegure un liderazgo efectivo ${ }^{2,3}$.

Según la conducta del líder, el liderazgo puede ser autocrático, democrático o liberal, ${ }^{2}$ pero también existe el estilo paternalista, mediador y participativo ${ }^{3}$. Por otro lado, si es la situación la que determina el liderazgo, entonces los rasgos y las conductas no serían el elemento principal que debería analizarse. Ello se debe a que, si las circunstancias cambian, el líder debe adaptar su conducta a los requerimientos y las demandas de cada situación en particular ${ }^{3}$. Otras teorías situacionales tratan de la relación entre efectividad y personalidad del líder, interacción entre el líder y la madurez de los trabajadores, así como la determinación de metas y su relación con la motivación y la eliminación de obstáculos².

Por su parte, las teorías contemporáneas proponen un acercamiento integral y multidireccional, analizando factores como la tarea, el tipo de organización, la estructura de trabajo, el presupuesto y el cambio organizativo requerido 2 . Todas ellas enfatizan que cada patrón de conducta puede ser efectivo en diversas situaciones, pero no es óptimo para la resolución de todas ellas ${ }^{3}$. Entre estas teorías se encuentran el liderazgo transaccional y el liderazgo transformacional, los cuales se describen a continuación.

\section{El liderazgo transaccional y transformacional en las instituciones de salud}

El liderazgo transaccional se basa en el intercambio o la transacción. En este, el líder utiliza su poder para recompensar o sancionar a sus subordinados, y no muestra interés en el desarrollo organizativo. Su ejercicio se centra en especificar las labores que desarrollará cada uno de los trabajadores y en verificar que estas se hagan del modo adecuado. Se caracteriza por dos subdimensiones: 1) la «recompensa contingente», según la cual el líder determina la recompensa o la sanción dependiendo del cumplimiento de objetivos organizacionales; y 2) la «administración por excepción", en la que el líder interviene únicamente para corregir acciones y asegurar el 
cumplimiento de metas de la organización. En este tipo de liderazgo, la mayoría de sus acciones son correctivas y sus críticas generalmente son negativas ${ }^{4}$.

En contraparte, el liderazgo transformacional concibe al líder como agente de cambio, como principal promotor del compromiso y motivador del trabajo en equipo. Busca también el significado de sus labores, y su ejercicio se basa en valores ${ }^{4}$. Este tipo de líder motiva a sus colaboradores a compartir su visión y a lograr las metas que este mismo dicta ${ }^{5}$.

Entre sus rasgos de personalidad, el líder transformacional es carismático, personalista, seguro de sí mismo y con una alta influencia sobre los demás ${ }^{2}$. Este tipo de liderazgo es muy útil en empresas con un alto propósito ideológico y en situación de tensión 0 de incertidumbre ${ }^{3}$. Es el liderazgo que aporta mayores beneficios a las instituciones de salud, ya que permite una visión compartida y promueve el compromiso, la cooperación, la lealtad y la innovación continua. También permite a los integrantes de la organización no solo el desarrollarse en el ámbito organizacional, sino también intelectualmente. Ello facilita la toma de decisiones, promueve la delegación de tareas y permite una mayor autonomía de los colaboradores ${ }^{6}$. Sin embargo, la principal desventaja de este estilo de liderazgo es que se requiere mucho tiempo para crear un clima adecuado de interacción y de confianza entre colaboradores y directivos ${ }^{4}$.

\section{Las instituciones de salud como empresas públicas y el liderazgo}

El Sistema Nacional de Salud (SNS) cuenta con tres niveles de atención: servicios básicos de salud otorgados en centros de salud y clínicas de medicina familiar (primer nivel), servicios de atención hospitalaria con consulta externa de especialidades básicas (segundo nivel), y hospitales de alta especialidad, centros médicos nacionales y hospitales de referencia (tercer nivel); estos últimos se caracterizan por tener un alto financiamiento y tecnología de punta ${ }^{7}$.

En todos los niveles de atención el personal es heterogéneo y está integrado en su mayoría por médi$\cos$ y enfermeras, entre otros profesionales de la salud, personal administrativo y de servicios. Dependiendo de la complejidad de la institución, la plantilla de personal va de unas cuantas decenas hasta miles de personas ${ }^{7}$. De este modo, liderar instituciones de salud es en realidad liderar verdaderas empresas. Los hospitales como empresas públicas poseen metas específicas derivadas de sus programas de salud, las cuales deben cumplirse con economía y eficiencia en respuesta a las necesidades de la población8.

Por lo anterior, las situaciones que diariamente enfrenta el líder son cada vez más complejas. Ello obliga a la ADIS a buscar nuevos enfoques de liderazgo que permitan mayor eficacia, mejor eficiencia y más productividad. El nuevo líder en salud debe caracterizarse por tomar decisiones imprevistas con una alta asertividad, poseer un pensamiento creativo e innovador, ser colaborativo, abierto al aprendizaje y al cambio, promover el desarrollo organizativo, poseer una visión sistémica y estratégica, ser congruente, ser motivador, capaz de influir en los demás y con una alta capacidad resolutiva ante las contingencias ${ }^{2}$.

Erróneamente, se piensa que la eficiencia de la atención en salud (atender pacientes con el mínimo de recursos) se logra reduciendo el personal o asegurando la compra de insumos con un menor costo. Si bien con ello se logrará liberar recursos, lo anterior sacrificaría la calidad de la atención. La gestión administrativa en salud debe resolver las contingencias con lo mínimo necesario, sin derroche, pero también sin negar recursos. Prevenir la enfermedad, las complicaciones, la discapacidad y la mortalidad es siempre la acción más sostenible en la economía de un país.

Por lo anterior, en las instituciones de salud es necesario modernizar la gestión para hacer eficiente el gasto público y elevar la calidad de los servicios. La administración de organizaciones tanto públicas como privadas debe ser capaz de liderar en entornos complejos, cambiantes e inciertos, con unos líderes que sean flexibles y capaces de interpretar las señales del entorno en beneficio de sus organizaciones, con una velocidad de respuesta a los cambios adecuada, con eficiencia, equidad y visión empresarial. Por tanto, los hospitales con una buena rentabilidad son por ende autosostenibles ${ }^{9}$.

\section{La alta dirección y los problemas de liderazgo en las instituciones de salud}

Aunque todas las instituciones de salud tienen puntos comunes entre sí, cada una de ellas debe ser liderada y administrada de modo particular. Aun realizando el mismo tipo de actividad, no existen dos hospitales o clínicas iguales; cada cual tiene su estructura, dinámica y cultura organizativa propia ${ }^{10}$. Por ello, dirigir las instituciones de salud sin tomar en 
cuenta sus peculiaridades y su entorno sería probablemente el principal error de gestión de un líder y un preámbulo para el caos.

Liderar es influir de alguna manera sobre los colaboradores para llevar a cabo las metas de la organización $^{11}$. En salud, el liderazgo permite mejorar la prestación del servicio en el contexto institucional en particular, lo que favorece el cambio organizativo para el beneficio de la población usuaria ${ }^{12}$.

El liderazgo es la habilidad directiva más importante, promueve el trabajo en equipo y es un factor clave para el desarrollo de la organización ${ }^{12}$. Su ejercicio adecuado permite cinco elementos fundamentales para la institución: 1) visión; 2) identificación; 3) utilización de habilidades complementarias de otros; 4) compromiso; y 5) aprendizaje organizativo. El liderazgo es también una fortaleza que eleva la calidad de los servicios de salud, así como la eficacia de la organización. Sin embargo, la relación de estos con el grado de experiencia del líder aún debe investigarse ${ }^{13}$.

En las instituciones de salud, cuando el profesional de la salud ocupa un cargo directivo pueden presentarse dos escenarios: que no posea la experiencia necesaria para liderar y administrar la institución o que, poseyéndola, no conozca la cartera de servicios ni los programas de salud que lidera. Las anteriores situaciones provocan un liderazgo ineficaz y una mala o deficiente administración, lo que se traduce en un pobre seguimiento del líder, mal ejercicio del poder, falta de planeación estratégica, poca sensibilidad hacia las necesidades del personal, poca capacidad resolutiva y, por ende, una alta rotación de directivos.

En consecuencia, si el problema antes mencionado se presenta en las instituciones de salud, se puede inferir que el líder no se encuentra preparado para ejercer sus funciones administrativas. Algunas de las causas podrían ser las siguientes: no tiene una idea definida de su función ni de lo que se espera de él; no centra su ascenso jerárquico en la capacitación continua; prioriza más la imagen y el prestigio que la adecuada realización de sus funciones; centra su administración en el ejercicio de la autoridad; duplica actividades; no prioriza las necesidades; se conforma con resultados mediocres o modestos; es inseguro al tomar decisiones o las toma por intuición; posee una mala capacidad de reacción a las crisis; posee un mal manejo de la frustración; presenta una conducta agresiva administrativa; o promueve la división organizativa ${ }^{8}$.

La ADIS tiende a la frustración cuando se enfrenta a situaciones administrativas en las cuales no posee ninguna experiencia. Ello puede traer como consecuencia que excelentes médicos se perfilen como malos e insatisfechos directivos. Cuanto mayor sea el ascenso en la jerarquía hospitalaria, mayor es su desvinculación de la praxis clínica, lo que provocará un decremento en la vigencia de sus conocimientos y puede contribuir a incrementar el grado de insatisfacción, disminuyendo aún más el interés en el ejercicio de su liderazgo ${ }^{14}$.

Es importante mencionar que en las instituciones de salud no existe una estructura rígida identificable de liderazgo. La visión es particular de cada institución o, simplemente, no existe. Ello deriva en que cada líder implemente su propio estilo de dirección y establezca su propia escala de prioridades y valores $^{15}$. De este modo, un profesional de la salud sin capacitación administrativa ejecutará una débil planificación y conducción de los procesos al interior de las instituciones, además de una escasa utilización de los recursos técnicos, por lo que se limita su efectividad. También es frecuente observar el poco o nulo apego a los procesos que él mismo establece, desorganización y desarticulación en la cooperación con los colaboradores, y resultados tan modestos que apenas son visibles ${ }^{15,16}$.

Tal vez una de las fallas recurrentes en la gestión sanitaria es una débil o ausente influencia generalizada en la organización, lo que provoca una difusa visión organizativa y, por lo tanto, no logra concretarse el trabajo en equipo. Un déficit de liderazgo también genera un pobre estímulo intelectual para los trabajadores, se incentiva poco la solución de problemas mediante la innovación, y un nulo e ineficiente contacto humano con un detrimento de las relaciones interpersonales ${ }^{16,17}$. En cuanto a la función de control, se evidencia una carencia de gestión basada en objetivos, desvinculación entre lo programado y lo realizado, ausencia de flujo de información que retroalimente la toma de decisiones, y una imprudente atención de los problemas organizativos detectados ${ }^{18}$.

Otro de los errores observados en la ADIS es el desconocimiento de las instituciones que lideran. Si el directivo no conoce qué motiva a sus colaboradores, no tendrá la capacidad de influir en su conducta y administrará erróneamente los incentivos. Elegir erróneamente los incentivos para el personal trae como consecuencia que estos sean ineficaces para algunos e incluso contraproducentes para otros ${ }^{14}$.

En cuanto al ejercicio del poder, se ha observado que un abuso del estilo autoritario provoca una menor aceptación y poco respaldo por parte de los colaboradores. Sin embargo, tampoco el ejercicio de un 
estilo exclusivamente democrático asegura que el directivo sea concebido y aceptado como líder. En este último, son frecuentes las actitudes permisivas, lo que puede llegar a comprometer seriamente el cumplimiento de metas. Una gestión basada en el permisivismo necesariamente traerá caos a la organización. Tanto el autoritarismo como la excesiva permisibilidad son factores que afectan y limitan el establecer y desarrollar relaciones interpersonales satisfactorias, así como el seguimiento del líder por parte de sus colaboradores ${ }^{19}$.

El papel del liderazgo en el sano funcionamiento de una organización es fundamental. La habilidad del líder para inspirar, motivar y crear compromiso con los objetivos comunes es crucial. Un líder verdadero gestiona las motivaciones, los valores y las emociones propias y ajenas; genera consciencia y prioriza el beneficio común antes que el personal20,21.

\section{Es prioritario articular la teoría del liderazgo con el desarrollo del directivo}

Los sistemas de salud se encuentran inmersos en entornos dinámicos e inciertos. Requieren líderes flexibles, proactivos y prospectivos que tengan una gran capacidad de flexibilidad y adaptabilidad para adecuarse a las exigencias del medio. Sin duda alguna, la habilidad de liderar y administrar deberían ser habilidades enseñadas, adquiridas y perfeccionadas durante la formación, desde el pregrado hasta el posgrado.

Investigar con mayor profundidad en esta área permitirá determinar el modo de evaluar adecuadamente el liderazgo en salud, si se tienen en cuenta las habilidades directivas del clínico para su propuesta administrativa, su efectividad en los puestos de responsabilidad, el papel del liderazgo en la consecución de objetivos estratégicos y metas, y cuáles son los criterios para considerar a un buen clínico como buen administrador y líder ${ }^{22}$.

Una alta proporción de directivos aprenden sobre la marcha, por ensayo y error, de tal modo que un líder se hace efectivo en el grado en que logre el autoconocimiento, se capacite, entrene y aprenda de sus experiencias ${ }^{23}$. A este respecto, es necesario considerar que es tan riesgoso para la institución que un médico sin experiencia administrativa asuma una alta jerarquía como que un administrativo no profesional de la salud pretenda atender pacientes. Por ello, se vuelve absolutamente necesario articular la teoría con la práctica mediante la profesionalización y el desarrollo del liderazgo de la ADIS, ya que esto permite definir el futuro de la institución, conducir a los colaboradores de acuerdo con su visión e inspirarles a hacerla realidad. Por lo tanto, el líder en salud debe crear una visión compartida, mejorar los procesos, facilitar el aprendizaje para la acción, marcar la ruta del cambio a seguir y alentar el ánimo de los colaboradores ${ }^{23,24}$.

\section{Un marco para mejorar el liderazgo en las instituciones de salud}

Sin pretender dar «explicaciones mágicas» ni «recetas de cocina", este texto sugiere algunos aspectos para mejorar el ejercicio del liderazgo en las instituciones de salud. La administración y el liderazgo son un arte; no se trata de trabajar todo a la vez, sino que se debe priorizar y gestionar los cambios de modo gradual y progresivo, ser prudente como para no «asustar» a la organización, pero no tan lento que se piense que «no sucede nada». Todo cambio deriva del aprendizaje y, para ser significativo, debe «significar algo" para aquellos que lo experimentan.

Existen diversas fuentes para «apalancar» el cambio a un liderazgo más efectivo que es necesario señalar:

- Administración de sistemas complejos. Los hospitales deben entenderse como un organismo complejo con múltiples interacciones de sus subsistemas, con relaciones dinámicas y no del todo predecibles. Es importante enfatizar que la elección correcta de las variables de cambio, por pequeñas que estas sean, puede provocar enormes resultados 25,26 .

- Gestión por objetivos estratégicos y basada en el desempeño. Una gestión hospitalaria sin datos duros se convierte en una toma de decisiones basada en la serendipia, en un asunto anecdótico con valoraciones sustentadas en juicios cualitativos. No obstante, no dejan de ser experiencias; las valoraciones basadas en la subjetividad no aportan un sustento sólido a la gestión, como son el cumplimiento de objetivos estratégicos y la evaluación del desempeño de líderes y empleados por igual ${ }^{25,27}$.

- Gestión de la experiencia colectiva. Como ya se ha comentado, la implementación de un sistema hospitalario de calidad total es un asunto de aprendizaje. Desgraciadamente, la experiencia del cambio se enfoca a los empleados de las diversas instituciones de salud en lugar de iniciar 
por la ADIS y de ahí transformar las instituciones. Las crisis afectan a todos, y a todos les corresponde resolverlas. Por ello, el cambio es un asunto de todos los miembros de la organización y no solo de unos cuantos ${ }^{25,28}$.

- Investigación, desarrollo e innovación (I+D+i). Es bien sabido que, en las instituciones de salud, muchas de las experiencias de éxito no se publican, no se incentiva la investigación o no se logra vincular esta con las necesidades reales en salud del país o de la localidad donde se encuentra el hospital. No se promueven el desarrollo de patentes ni el registro de la propiedad intelectual. Además, existe una inercia al cambio que los clínicos con o sin experiencia administrativa pocas veces logran sortear y superar. De este modo, lo que brindaría una enorme ventaja competitiva a las instituciones de salud se convierte en su principal debilidad y termina por ser su mayor amenaza en un mundo globalizado ${ }^{25,29}$.

- Gestión del capital humano. La clave del liderazgo es no olvidar que los trabajadores no son máquinas, sino personas. De las personas dependen la productividad, la eficacia y la eficiencia; también asumir y practicar los valores de organización y personales. Es importante enfatizar que los intereses de la organización no están peleados con los intereses del trabajador de la salud; lograr que ambos se alienen es un arte para el líder de instituciones de salud. Hoy día, las instituciones son más competitivas, exigen nuevos niveles de compromiso, de asumir los hechos y responder adecuadamente a las diversas crisis que se presentan en la gestión hospitalaria, tomar mejores decisiones y transformar los hospitales en empresas más humanas. Todos estos aspectos no podrían lograrse sin considerar el aspecto humano de la administración ${ }^{25,30}$.

\section{Conclusiones}

Los problemas de liderazgo son un fenómeno frecuente en la ADIS. En su gran mayoría, estos se encuentran relacionados con la poca o nula experiencia administrativa del directivo, o con el desconocimiento de los programas de salud que lidera. Entre los principales problemas de liderazgo identificados se encuentran la falta de visión empresarial, una pobre identificación del líder con los colaboradores, un deficiente compromiso con la organización, el desconocimiento de la dinámica de la institución que se lidera, una deficiente planeación estratégica, una mala toma de decisiones, un mal ejercicio del poder y el conformarse con resultados subóptimos.

Derivado del mal ejercicio del liderazgo, el directivo puede volverse inefectivo, desconocer la trascendencia de sus funciones, depreciar la capacitación continua, frustrarse por no tomar decisiones asertivas y mostrar un profundo desinterés por la excelencia de la organización. Esto provocará en los colaboradores una pobre identificación con el líder, falta de motivación hacia el logro de metas y hasta rechazo a los directivos. Lo anterior trae como consecuencia una alta rotación de directivos y un incremento en la incertidumbre y la tensión organizativa en las instituciones de salud.

El liderazgo no es innato, es una habilidad que puede ser desarrollada. Es factor clave en el desarrollo de las funciones administrativas y su adecuado ejercicio incrementa la calidad de la atención y la eficacia de los colaboradores en el cumplimiento de las metas de la organización. Si la ADIS no posee la experiencia necesaria, esta debe profesionalizarse; si no posee el conocimiento de los planes estratégicos en salud que lidera, debe buscar conocerlos en profundidad. Que el directivo identifique los problemas de liderazgo de su administración le permitirá redefinir el problema como una oportunidad de mejora para una adecuada práctica administrativa.

El líder actual en las instituciones de salud debe ser crítico, creativo e innovador, y centrarse en identificar los problemas antes de que se produzcan y actuar antes de las consecuencias. Tiene que ser el diseñador de una estrategia sólida con una visión definida y compartida, con una alta capacidad de influencia sobre los demás, y ser reconocido como agente de cambio.

\section{Conflicto de intereses}

Los autores declaran no tener conflictos de intereses.

\section{Bibliografía}

1. Romolerux de Mena E. Liderazgo y éxito organizacional. Polémika. 2010;1:156-63.

2. Aguirre $H$, Mazón B. Calidad y liderazgo en medicina. Revista CONAMED. 2013;18:172-82

3. Ganga F, Navarrete E. Enfoques asociados al liderazgo eficaz para la organización. Gaceta Laboral. 2013;19:52-77.

4. Almirón V, Tikhomirova A, Trejo A, García J. Liderazgo transaccional vs liderazgo transformacional. Reidocrea. 2015;4:24-7.

5. Avolio B, Walumbwa F, Weber T. Leadership: current theories, research, and future directions. Annu Rev Psychol. 2009;60:421-49.

6. Hernández T, Corichi A, García M, Martínez E, Fuentes B. Liderazgo transformacional del personal médico en institución pública del sector salud. Administración para el Desarrollo. 2015;9:21-36. 
7. Burr C, Piñó A, Quiroz L, Martín E. Por los caminos del Sistema Nacional de Salud. En: Guía para el paciente participativo. Atrévete a saber. Atrévete a exigir. Editorial Mexicana. México, D.F., México, 2017.

8. Pavón P, Gogeascoechea M. La importancia de la administración en salud. Rev Med UV. 2004:4:13-6.

9. García C. El hospital como empresa: nuevas prácticas, nuevos trabajadores. Universitas Psychologica. 2007;6:143-54

10. Hervás F. El hospital empresa y la sanidad asistencial. Calidad y rentabilidad. México: Díaz de Santos; 2007.

11. National Minority AIDS Council. Desarrollo del liderazgo. Washington, DC: NMAC; 2003

12. Pinzón O. Liderazgo en los servicios de salud. Rev CSV. 2014;6:37-53.

13. Castañeda O. El papel del liderazgo en la gestión médica. Aten Fam. 2015;22:1-2.

14. Valor J, Ribera J. Gestión en la empresa hospitalaria. Navarra: IESE Business School; 1990

15. Colindres $\mathrm{H}$. Fortaleciendo el liderazgo y gerencia de instituciones de salud en procesos de reforma. Guatemala: Management Sciences for Health; 2004.

16. Hernández T, Duana D. Estudio diagnóstico de liderazgo transformacional en personal de enfermería que labora en hospitales públicos mexicanos. Rev CIMEXUS. 2018:13:89-109.

17. Danet A, García I, March J. Liderazgo transformacional en las organizaciones sanitarias. Rev Comunicación Sal. 2016;6:81-95

18. Barea J. El hospital, empresa de servicios. Revista Fundación Signo. 2000;1:93-100.

19. Casales J. Patrones de liderazgo, estrés y desempeño profesional en instituciones de salud. Rev Cubana Psicol. 2000;17:131-46.
20. Hermosilla D, Amutio A, Costa S, Páez D. El liderazgo transformacional en las organizaciones: variables mediadoras y consecuencias a largo plazo. Rev Psicol Trab Organ. 2016;32:135-43.

21. Aguilar MC, Calvo A, García MA. Valores laborales y percepción del estilo de liderazgo en personal de enfermería. Sal Publ Mex. 2007:49:401-7.

22. Ayuso D, González M. Liderazgo sanitario. En: Ayuso D, Herrera I, editores. El liderazgo en los entornos sanitarios. Formas de gestión. Díaz de Santos. Madrid, España, 2017.

23. Sharma MK, Shilpa J. Leadership management: principles, models and theories. Glob J Manag Bus Stud. 2013;3:309-18.

24. Carrada T. Liderazgo médico en sistemas de salud. Diseño de un nuevo modelo de gestión. Rev Mex Patol Clin. 2003:50:142-56.

25. Reinertsen J, Bisognano M, Pugh M. Seven leadership leverage points for organization-level improvement in health care. Cambridge, Massachusetts: Institute for Healthcare Improvement; 2008.

26. Camargo M, Van der Berghe E, Eliecer J, Gómez N. Ingeniería de sistemas complejos y logística hospitalaria. Modelamiento y simulación de la dinámica de aseo en el Hospital Vista Hermosa I Nivel. Bogotá, D.C.: Universidad del Rosario, Facultad de Administración; 2013.

27. Riaño M, García J. Gestión estratégica para hospitales universitarios. Rev Fac Med. 2016;64:615-20.

28. Sáenz L. Modernización de la gestión hospitalaria colombiana: lecciones aprendidas de la transformación de los hospitales en empresas sociales del Estado. PAHO: LACRSS; 2001

29. Palencia F, García J. Innovación e investigación en hospitales universitarios. Rev Fac Med. 2016;64:741-7.

30. Delgado N, Delgado D. El líder y el liderazgo: reflexiones. Rev Interam Bibliotecol. 2003;26:75-88. 